\title{
Top Production from Black Holes at the LHC
}

\author{
M. Mohammadi Najafabadi 1 and S. Paktinat Mehdiabadi 2 \\ School of Particles and Accelerators, \\ Institute for Research in Fundamental Sciences (IPM) \\ P.O. Box 19395-5531, Tehran, Iran
}

\begin{abstract}
In theories with large extra dimension and with low quantum gravity scale near a $\mathrm{TeV}$, it is expected that $\mathrm{TeV}$-scale black holes to be produced in proton-proton collisions at the LHC with the center of mass energy of $14 \mathrm{TeV}$. Since the black holes temperature can be around $1 \mathrm{TeV}$, top quark production is expected from them via Hawking radiation. Within the Standard Model of particle physics top quarks are produced via strong interaction in $t \bar{t}$ pairs or via electroweak interaction singly. Therefore, black holes can be the new source of top quark production. In this article we present the total cross sections and transverse momentum distributions of top quark production from black holes at the LHC. We find that the top quarks from black holes tend to reside at very high transverse momentum region so it can be a very useful signature for the black holes at the LHC.
\end{abstract}

\footnotetext{
${ }^{1}$ Email: mojtaba@ipm.ir

${ }^{2}$ Email: paktinat@ipm.ir
} 


\section{Introduction}

One of the most exciting possiblities for new physics at the LHC is the production of black holes which can occur in theories with large extra dimensions. As the black hole masses at the LHC are relatively small and the temperatures of black holes are very high (around $1 \mathrm{TeV}$ ), they will decay quickly through Hawking radiation into some thermally distributed particles of the Standard Model particles (before fragmentation of the emitted partons). This produces a signature unlike all other new predicted effects. The black hole evaporation's process connects quantum gravity with quantum field theory and particle physics. It is a promising way towards the understanding of the Planck scale physics [1].

Because of the large mass of the top quark among all other observed particles within the Standard Model, it plays a special role in the generation of masses and Electroweak Symmetry Breaking (EWSB). Therefore, it is crucial to study its interactions with other particles and to know all possible sources of top quark production. At the LHC, top quarks can be produced in $t \bar{t}$ pairs via strong interactions through two subprocesses: gluon-gluon fusion $(g g \rightarrow t \bar{t})$ and quark-antiquark annihilation $(q \bar{q} \rightarrow t \bar{t})$. The total cross section of top pair prodution at the LHC is $830 \mathrm{pb}$. Another source of top quark at the LHC is single top quark production. Single top quarks are produced via the $t$-channel $\left(b q \rightarrow q^{\prime} t\right)$, the $s$-channel process $\left(q \bar{q}^{\prime} \rightarrow t \bar{b}\right)$ and the $t W-$ channel process $(g b \rightarrow t W)$. The $t$-channel is the largest source of single top quark production with the cross section of $245 \mathrm{pb}$ and the $s$-channel is the smallest with the cross section of $10 \mathrm{pb}$. The cross section of $t W$-channel is $60 \mathrm{pb}$. Table 1 presents the cross sections and the predicted relative statistical uncertainties on measurement of the cross sections of different top production processes. These results have been derived using full CMS detector simulation at the LHC $[2]$.

In this paper we compare top quark production cross sections from TeV scale black hole production at the LHC via Hawking radiation with the cross section of top quark production from Standard Model which were mentioned above. We also compare the transverse momentum differential cross section $\left(\frac{d \sigma}{d p_{T}}\right)$ of the top quarks coming from black holes with the transverse momentum distribution of single top quarks. 


\begin{tabular}{|c|c|c|c|c|}
\hline Process & $t$-ch & $s$-ch & $t W$-ch & $t \bar{t}$ \\
\hline Cross section & $245 \mathrm{pb}$ & $10 \mathrm{pb}$ & $60 \mathrm{pb}$ & $833 \mathrm{pb}$ \\
\hline Statistical uncertainty $\left(\frac{\Delta \sigma}{\sigma}\right)$ & $2.7 \%$ & $17.6 \%$ & $9.2 \%$ & $0.4 \%$ (lepton+jets) \\
\hline
\end{tabular}

Table 1: The cross sections and the predicted statistical uncertainties on the cross sections of different top quark production processes by the CMS detector at the LHC for $10 \mathrm{fb}^{-1}$ of integrated luminosity.

\section{Top Quark Production from a Black Hole Evapo- ration}

To simulate the production and decay of black holes at the LHC, Charybdis1.003 [3] package is used. The program is interfaced, via the Les Houches accord [4, to HERWIG6.510 [5] to perform the parton shower evolution of the partons produced in the decay and their hadronization. As for the proton parton distribution functions (PDF), MRSD_' [6] is used. The $Q^{2}$ scale taken to be equal to $M_{B H}$, which is within the allowed range for this PDF set, up to the LHC kinematic limit. The dependency of the cross section on the choice of the PDF is in the level of $10 \%$. Different parameters are set to the default values of the programs unless it is mentioned explicitly. The default value for top quark mass is $174.3 \mathrm{GeV} / c^{2}$. For comparison $m_{t}=170 \mathrm{GeV} / c^{2}$ was also tried. The largest difference was seen for $t \bar{t}$ production which was $10 \%$.

To study the top quark production from black holes, we only consider the events that have only one (anti)top or only one pair of $t \bar{t}$. Events with other combinations of top quarks (same sign top quarks) are rejected from the analysis. One can study these events by looking for two or more same sign leptons. Figure 1 shows the distribution of the $\frac{d \sigma}{d M_{B H}} \times B R$ versus the black hole mass for single top (top row) and $t \bar{t}$ (bottom row) production for different values of the Planck mass and total number of dimensions ( $\mathrm{d}=4$ + number of the extra dimensions). It is seen that the cross section times branching ratio is more sensitive to Planck mass than the number of the extra dimensions. In particular, for single top changing the number of extra dimensions doesn't affect the results. As can be seen from Figure 1 the cross sections decreases rapidly when both the Planck scale and 

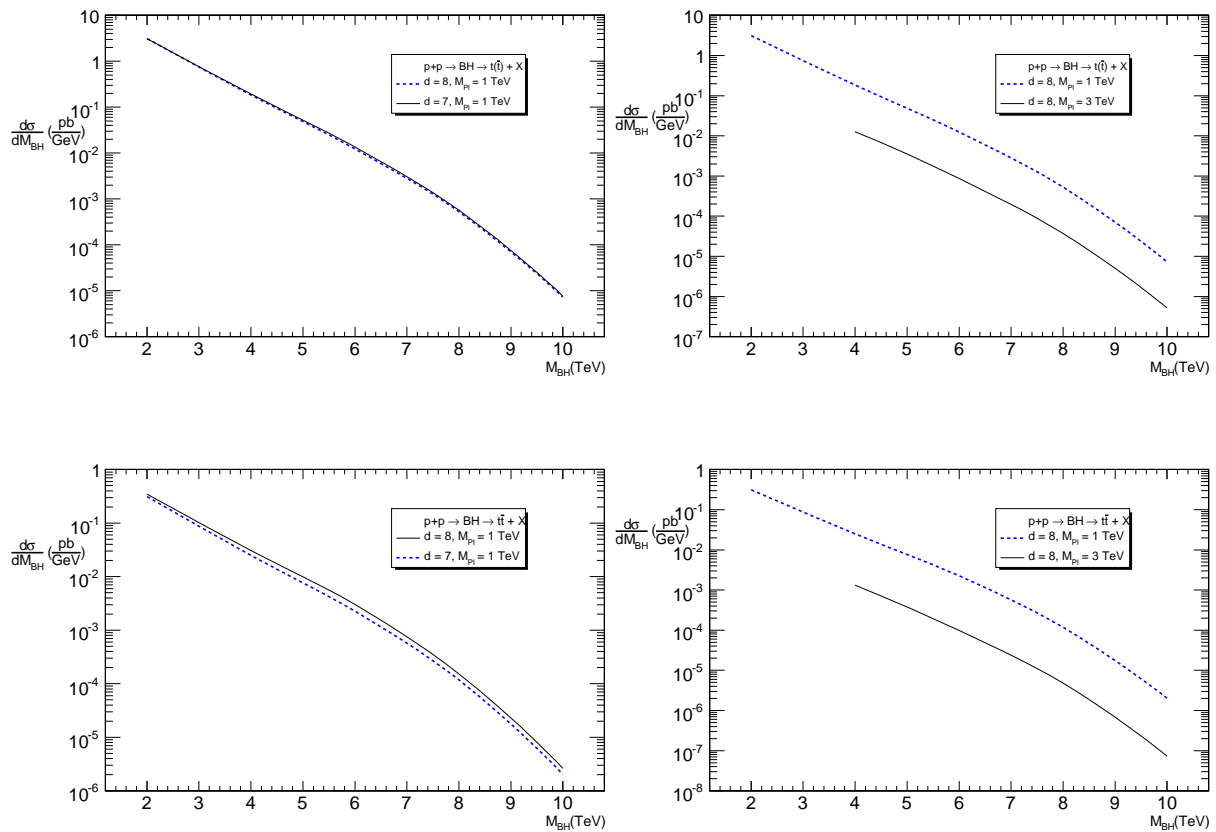

Figure 1: $\frac{d \sigma}{d M_{B H}} \times B R$ versus the black hole mass for single top (top row) and $t \bar{t}$ (bottom row) at the LHC.

black hole mass increase.

If the fundamental Planck mass is $1 \mathrm{TeV}$, the total cross section times branching ratio for single top and $t \bar{t}$ production is respectively $10 \mathrm{nb}$ and $1 \mathrm{nb}$ which are very large numbers comparing to the Standard Model predictions for these channels. As mentioned in Table 1, the LHC is able to measure the cross sections of $t \bar{t}$ and single top very precisely. Therefore, new source of top quark such as black holes can be observed at the LHC experiments.

Figure 2 shows the distribution of the transverse momentum of the top quarks from SM single top quark (t-channel) and single top quarks from the decay of a black hole. The specifications of the black hole are shown in the figure. The figure only compares the shapes of the distributions. It is clear that top quarks from the decay of black holes are much harder than the predicted top quarks in Standard Model. According to Figure 2, the transverse momentum distribution of single top quarks from Standard Model has a mean value of $66.7 \mathrm{GeV}$ while the mean value of the transverse momentum distribution of single top quarks produced from black holes with a mass of $2 \mathrm{TeV}$ and number of extra 


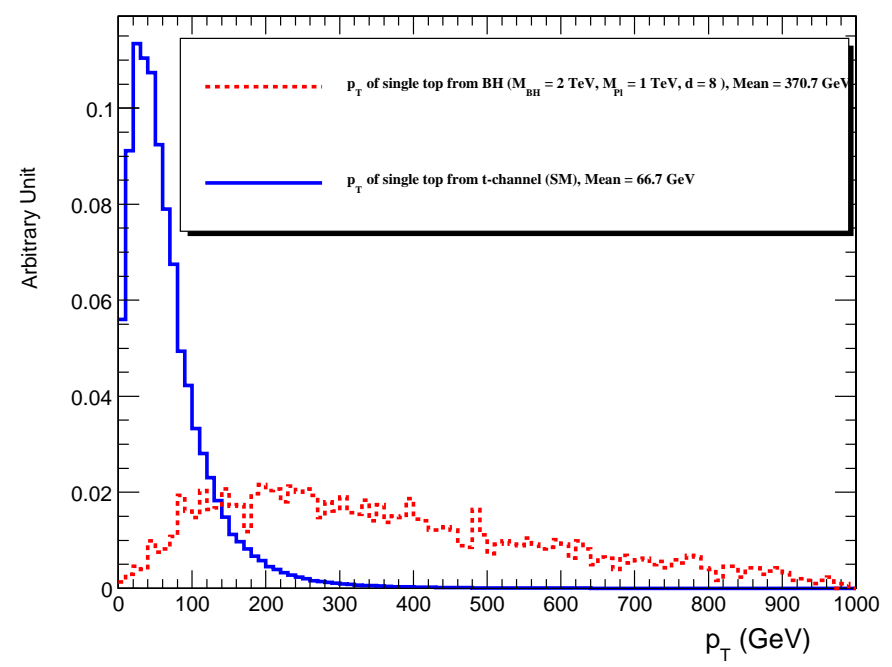

Figure 2: The transverse momentum distribution $\left(P_{T}\right)$ of the top quarks from SM single top quark (t-channel) and single top quarks from the decay of a black hole are compared.

dimensions of four is around $370.7 \mathrm{GeV}$. This feature can be used to discover the black holes and distinguish the top quarks from different sources.

\section{Conclusion}

The top quark production from the decay of a black hole was considered for different values of the free parameters. It was shown that if the black holes are produced at the LHC, an unnegligible excess in the number of the produced top quarks can be seen. If the fundamental Planck mass is very high, the cross section for top quark production decreases significantly, but the produced top quarks are much harder than those from the Standard Model, so studying the high transverse momentum top quarks can indicate the presence of the signal.

\section{Acknowledgments}

The authors would like to thank M. Alishahiha for reading the paper and raising the useful comments. M. Mohammadi is grateful to A. N. Khorramian for useful discussions. 


\section{References}

[1] N. Arkani-Hamed, S. Dimopoulos and G.R. Dvali, Phys. Lett. B429, 263 (1998); I. Antoniadis, N. Arkani-Hamed, S. Dimopoulos and G.R. Dvali, Phys. Lett. B436, 257 (1998); L. Randall and R. Sundrum, Phys. Rev. Lett. 83, 3370 (1999); S. Dimopoulos and G. Landsberg, Phys. Rev. Lett. 87, 161602 (2001); G.C. Nayak, J. Smith, Phys.Rev. D74, 014007 (2006).

[2] CMS Collaboration, CMS PTDR, Vol.II, CERN/LHCC 2006-021, J. Phys. G34, 995 (2007).

[3] C.M. Harris, P. Richardson and B.R. Webber, JHEP 0308, 033 (2003), hep-ph/0307305; C.M. Harris, P. Kanti, JHEP 0310, 014 (2003), hep-ph/0309054.

[4] P. Skands, B.C. Allanach, H. Baer, C. Balzs, G. Blanger, F. Boudjema, A. Djouadi, R. Godbole, J. Guasch, S. Heinemeyer, W. Kilian, J-L. Kneur, S. Kraml, F. Moortgat, S. Moretti, M. Mhlleitner, W. Porod, A. Pukhov, P. Richardson, S. Schumann, P. Slavich, M. Spira, G. Weiglein, JHEP 0407, 036 (2004), [hep-ph/0311123v3].

[5] G. Corcella, I.G. Knowles, G. Marchesini, S. Moretti, K. Odagiri, P. Richardson, M.H. Seymour, B.R. Webber, hep-ph/0210213] and refernces therein.

[6] A. D. Martin, W. J. Stirling and R. G. Roberts, Phys. Lett. B306, 145 (1993). 